\title{
Virologic and immunologic response to HAART, by age and regimen class
}

\author{
Keri N. Althoffa ${ }^{a}$ Amy C. Justice ${ }^{b}$, Stephen J. Gange ${ }^{a}$, Steven G. Deeks ${ }^{c}$, Michael S. Saag ${ }^{d}$, \\ Michael J. Silverberg ${ }^{\mathrm{e}}$, M. John Gill ${ }^{\mathrm{f}}$, Bryan Lau ${ }^{\mathrm{a}}$, Sonia Napravnik ${ }^{\mathrm{g}}$, Ellen Tedaldi ${ }^{\mathrm{h}}$, Marina \\ B. Klein', Kelly A. Gebo ${ }^{a}$, and the North American AIDS Cohorts Collaboration on \\ Research, Design (NA-ACCORD)
}

aJohns Hopkins University, Baltimore, Maryland bYale University and the VA Connecticut Healthcare System, New Haven, Connecticut 'University of California, San Francisco, San Francisco, California dUniversity of Alabama, Birmingham, Birmingham, Alabama ${ }^{\text {eKaiser }}$ Permanente Northern California, Oakland, California, USA fUniversity of Calgary, Calgary, Alberta, Canada IUniversity of North Carolina, Chapel Hill, Chapel Hill, North Carolina ${ }^{\text {hTemple }}$ University, Philadelphia, Pennsylvania, USA 'McGill University, Montreal, Quebec, Canada

\section{Abstract}

Objective-To determine the impact of age and initial HAART regimen class on virologic and immunologic response within 24 months after initiation.

Design-Pooled analysis of data from 19 prospective cohort studies in the North American AIDS Cohort Collaboration on Research and Design (NA-ACCORD).

Methods-Twelve thousand, one hundred and ninety-six antiretroviral-naive adults who initiated HAART between 1998 and 2008 using a boosted protease inhibitor-based regimen or a nonnucleoside reverse transcriptase inhibitor (NNRTI)-based regimen were included in our study. Discrete time-to-event models estimated adjusted hazard odds ratios (aHOR) and $95 \%$ confidence intervals (CIs) for suppressed viral load ( $\leq 500$ copies $/ \mathrm{ml}$ ) and, separately, at least 100 cells $/ \mu \mathrm{l}$ increase in CD4 cell count. Truncated, stabilized inverse probability weights accounted for selection biases from discontinuation of initial regimen class.

Results-Among 12196 eligible participants (mean age $=42$ years), 50\% changed regimen classes after initiation (57 and $48 \%$ of whom initiated protease inhibitor and NNRTI-based regimens, respectively). Mean CD4 cell count at initiation was similar by age. Virologic response to treatment was less likely in those initiating using a boosted protease inhibitor [aHOR $=0.77$ $(0.73,0.82)]$, regardless of age. Immunologic response decreased with increasing age [18-<30: ref; 30-<40: aHOR $0.92(0.85,1.00) ; 40-<50:$ aHOR $=0.85(0.78,0.92) ; 50-<60:$ aHOR $=0.82$ $(0.74,0.90) ; \geq 60$ : aHOR=0.74 $(0.65,0.85)]$, regardless of initial regimen.

Conclusion-We found no evidence of an interaction between age and initial anti-retroviral regimen on virologic or immunologic response to HAART; however, decreased immunologic response with increasing age may have implications for age-specific when-to-start guidelines.

(C) 2010 Wolters Kluwer Health | Lippincott Williams \& Wilkins

Correspondence to Dr Kelly A. Gebo, Johns Hopkins University School of Medicine, 1830 E. Monument Street, Room 435, Baltimore, MD 21287, USA. Tel: +1 410502 2325; fax: +1 410955 7889; kgebo@jhmi.edu.

Executive Committee: Richard D. Moore, Michael S. Saag, Stephen J. Gange, Mari M. Kitahata, Rosemary G. McKaig, Aimee

Freeman

Epidemiology/Biostatistics Core: Stephen J. Gange, Alison G. Abraham, Bryan Lau, Keri N. Althoff, Jinbing Zhang

Data Management Core: Mari M. Kitahata, Stephen E. Van Rompaey, Heidi M. Crane, Eric Webster, Liz Morton, Brenda Simon 


\section{Keywords}

age; CD4 lymphocyte count; HAART; HIV; viral load

\section{Introduction}

The proportion of Americans who are HIV-infected and older than 50 is growing. By 2015, more than $50 \%$ of all adults with HIV in the United States will be age at least 50 years [1,2]. It is believed this increasing proportion of HIV-infected individuals who are age at least 50 years is driven by increased survival owing to HAART [3]. New infections also contribute to the increase in HIV-infected adults aged at least 50 years, occurring at an estimated rate of 6.5 per 100000 population [2].

Although it is generally thought that older HIV-infected individuals initiating HAART will have a decreased immunological response [4-7,13-16] and an increased virologic response [4-12] compared with younger individuals, published data regarding immunologic and virologic response by age to HAART has been mixed. Some studies have demonstrated a smaller increase in CD4 cell count in older compared with younger adults [4-7,13-16], whereas others have been equivocal [10,12,17-19]. Some studies have demonstrated increased virologic suppression in older compared with younger adults [4-12] (which was hypothesized to be adherence-driven [5,20]), whereas one study reported the reverse [21], and many other studies have reported no differences [13,15-17,19]. Current guidelines recommend the initiation of HAART using either ritonavir-boosted protease inhibitors or nonnucleoside reverse-transcriptase inhibitors (NNRTI) [22,23]. These guidelines do not differentiate the recommendations by age, but there were a limited number of older individuals who contributed data to the studies that informed these guidelines. Of the participants in the largest randomized trial of initial HAART regimen class on HIV-1 RNA viral suppression, only $12 \%$ were age at least 50 years [24]. Similarly, the largest randomized trial of initial HAART regimen class on immunologic response had a median age of 38 years (interquartile range 32-44) [25].

Given the increasing number of individuals aged at least 50 years with HIV, it is important to determine if HIV antiretroviral guidelines should reflect age differences in virologic and immunologic response to HAART by regimen class. No study has evaluated immunologic and virologic response to HAART by both initial regimen class and age. Thus, the objective of this study was to evaluate the short-term virological and immunologic response to HAART by age and initial regimen class.

\section{Methods}

\section{Study population and design}

Owing to the large sample size needed to determine the interaction of age and HAART regimen class, our study was nested in the North American AIDS Cohort Collaboration on Research and Design (NA-ACCORD), a collaboration of eight interval and 13 clinical prospective cohort studies of demographically heterogeneous HIV-infected adults from the United States and Canada. The NA-ACCORD is a regional group of the International Epidemiological Databases to Evaluate AIDS (IeDEA) project with over 60 study sites in the United States and Canada. Details of the collaboration have been described elsewhere [26]. Briefly, contributing cohorts have used standardized methods of data collection and have submitted demographic, treatment, clinical, laboratory, and vital status data on enrolled participants. Each cohort's participation in NA-ACCORD was approved by the respective local institutional review boards. 
Nineteen NA-ACCORD cohorts contributed the necessary data and agreed to participate in this study. Participants who were at least 18-year-old from these cohorts were included in our study if they initiated HAART using a boosted protease inhibitor-based or NNRTI-based regimen in combination with two or more NRTIs between January 11998 and December 31 2008. Those initiating HAART using only nucleoside analogues, nonboosted protease inhibitors, or dual protease inhibitor-NNRTI regimens were excluded from the study. Individuals were required to have at least one follow-up visit; those who discontinued or changed regimens in the first month following HAART initiation were excluded. Further, all participants had a pre-HAART CD4 cell count and HIV-1 RNA measured at, or within 6 months prior to, HAART initiation. Lastly, to most appropriately examine the impact of the initial HAART regimen on our immunologic and virologic outcomes of interest, individuals with a record of treatment prior to HAART initiation, or an HIV-1 RNA at least 500 copies/ $\mathrm{ml}$ at HAART initiation were excluded.

\section{Outcomes: immunologic and virologic response}

Virologic and immunologic responses were measured up to 24 months following HAART initiation. Virologic response was measured using plasma HIV-1 RNA concentrations. Because of differences in HIV-1 RNA assays used in contributing cohorts over time, we defined an HIV-1 RNA measure of 500 copies/ml or less ('suppressed') as the virologic endpoint of interest. If a viral load result was reported as undetectable with an assay that used a lower limit of detection more than $500 \mathrm{copies} / \mathrm{ml}$ that measurement was discarded ( $<1 \%$ of all viral load measurements). Immunologic response was measured by CD4 cell count as reported by the cohorts. Time to an increase of 100 cells $/ \mu 1$ relative to CD 4 cell count at, or within 6 months prior to HAART initiation, was used in analyses as a clinically important response. If an individual had more than one HIV-1 RNA or CD4 cell count measurement within a month, the lowest HIV-1 RNA measurement and the arithmetic mean of the CD4 cell measurements were used.

\section{Exposures: age at HAART initiation and initial HAART regimen class}

Age at HAART initiation was calculated using the year of birth and the date of HAART initiation and was stratified into five groups $18-<30$ years, $30-<40$ years, $40-<50$ years, $50-$ $<60$ years and at least 60 years. HAARTwas defined as a regimen containing at least three antiretroviral drugs from two or more classes including a boosted protease inhibitor and/or NNRTI. Initial HAART regimen class was specified as the class used at initiation of HAART (boosted protease inhibitor or NNRTI).

\section{Other covariates of interest}

Demographic and variables related to HIV disease progression were measured as possible confounders. Demographic variables included sex and race (White, Black, Latino or other/ unknown). HIV transmission risk factor was categorized as men who have sex with men (MSM), history of injection drug use (IDU), heterosexual, or other/unknown. Individuals reporting IDU and either male-to-male sexual risk or heterosexual risk were classified as IDU. Other potential confounders included pre-HAART clinically diagnosed AIDS-defining illness (according to the 1993 criteria of the Centers for Disease Control and Prevention (CDC) excluding CD4 cell count less than 200 cells/ $\mu$ l), year of HAART initiation, sex and cohort. Data regarding time from HIV seroconversion and nadir CD4 cell count were not available.

\section{Statistical analysis}

Differences in means and proportions by age were tested using the chi-squared test statistics and ANOVA, respectively. 
Using observational data, we conducted analyses that mimic 'as-treated' analyses in clinical trials with imperfect compliance [27]. To accomplish this aim, we followed individuals from the time of HAART initiation in monthly intervals until the outcome of interest, 'regular' censoring consisting of the date of the last CD4 cell measurement, death, or December 31, 2008; or 'discontinuation' censoring reflecting the date discontinuation of, or switch from, the initial HAART regimen class.

Discontinuation censoring may induce a selection bias because only those individuals who do not discontinue or change from their regimen remain in the study population [28]. To correct this bias, weights were used to allow for influence of those who are censored owing to discontinuation, thus reducing the selection bias $[28,29]$. Details regarding the estimation and use of published methods [28] are provided in the Appendix,

http://links.lww.com/QAD/A71. Adjusted hazards odds ratios (aHOR) and 95\% confidence intervals were estimated for the initial HAARTregimen classes using weighted pooled logistic regression models with month as the interval of time. Kaplan-Meier estimates of the cumulative incidence of each outcome by age group were calculated, incorporating discontinuation and censoring weights [30]. To complement this analysis, we also conducted an 'intention-to-treat' analysis that did not censor at discontinuation or incorporate weights.

Analyses were performed using SAS 9.2 (Cary, North Carolina, USA) and a $P$-value of less than 0.05 was used to identify statistically significant variables.

\section{Results}

Nineteen NA-ACCORD cohorts participated in our study, contributing 116619 possible participants. Of these, 25449 participants initiated HAART with a boosted protease inhibitor-based or NNRTI-based regimen between January 1, 1998 and December 31, 2008. Eight thousand, one hundred and eighty-five participants were excluded owing to lack of HIV-1 RNA or CD4 cell measurement at, or within 6 months prior to, HAART initiation and 2903 participants were excluded owing to an HIV-1 RNA 500 copies $/ \mathrm{ml}$ or less at, or within 6 months prior to HAART initiation. Eleven participants were excluded because they were less than 18-years-old at HAART initiation. Finally, 1541 were excluded for failing to maintain their initial regimen for at least one month and 613 were excluded for not having at least one visit in 24 months after HAART initiation and prior to the date of administrative censoring (December 31 2008), resulting in 12196 eligible participants in our study population.

The mean age of the study population was 42 years (range 18, 83). The participants were predominantly male, and the proportion of males increased with increasing age (Table 1). The proportion of participants who had a history of IDU was greatest among the 40-59 yearold (21\%). Mean CD4 and HIV-1 RNA levels at HAART initiation ranged between 205250 cells $/ \mu 1$ and $4.66-4.73 \log _{10}$ copies $/ \mathrm{ml}$, respectively, across age groups. The proportion of adults with a clinical AIDS diagnosis prior to HAART ranged from 14-17\% across the age groups. Overall, $29 \%$ of participants initiated HAART on a boosted protease inhibitorbased regimen and $71 \%$ on an NNRTI-based regimen.

Half (50\%) (6134/12 196) of individuals did not remain on their initial therapy regimen class 24 months after initiation; 25\% (3100/12 169) changed regimen class and 25\% (3034/12 196) discontinued treatment. Of all the individuals who did not remain on their initial therapy regimen for 24 months after initiation, 43\% (2645/6134) changed regimen class or discontinued treatment in the first 6 months after HAART initiation. Those initiating HAART at the oldest ages had the smallest proportion that changed regimen classes or discontinued treatment $(18-<30$ years: $51 \% ; 30-<40$ years: $50 \% ; 40-<50$ years: $52 \%$; $50-$ 
$<60$ years: $50 \%$; $\geq 60$ years: $44 \%$; $P$-value $<0.01)$ and a greater proportion of individuals who initiated HAART using a boosted protease inhibitor-based regimen changed regimen class or discontinued treatment (57\%) compared with individuals initiating using an NNRTIbased regimen $(48 \%, P$-value $<0.01)$.

The cumulative incidence of HIV-1 RNA 500 copies/ml or less did not differ by age, even after stratifying by initial regimen (Fig. 1). The cumulative incidence of an increase of at least 100 cells/ $\mu$ differed by age with older individuals less likely to achieve this immunologic response (Fig. 2). The difference in immunologic response by age was seen among both boosted protease inhibitor and NNRTI initiators.

The results of the pooled logistic regression models are shown in Table 2. The occurrence of HIV-1 RNA suppression increased with increasing age, however, these estimates were not statistically significant. Immunologic response decreased with increasing age and the estimates were statistically significant. Those who initiated using a boosted protease inhibitor had a decrease in the hazard odds of virologic response compared with those who initiated using an NNRTI; additionally, there was not a significant increase in the likelihood of immunologic response on those initiating with boosted protease inhibitors compared with NNRTIs. Those with higher HIV-1 RNA levels at HAART initiation, Blacks, those in HIV risk groups other than MSM, and those initiating HAART in the late 1990s and early 2000s had a decrease in the hazard odds of HIV-1 RNA 500 copies/ml or less. Males, those with a lower HIV-1 RNA level at HAART initiation, those in HIV risk groups other than MSM, and those initiating HAART in the late 1990s and early-tomid 2000s had a decrease in the hazard odds of immunologic response.

The interaction of age and initial HAART regimen class was not statistically significant for our virologic outcome $(P=0.40)$ or immunologic outcome $(P=0.19)$. The increase in the hazard odds of viral suppression comparing NNRTI to boosted protease inhibitor was seen across all age groups (Table 3). The decrease in the hazard odds of immunologic response with increasing age was seen after stratification by initial regimen.

An intention-to-treat analysis that did not censor individuals at discontinuation or incorporate weights demonstrated similar findings, but, as expected, were attenuated. For example, those initiating on boosted protease inhibitors had a $21 \%$ decrease in the likelihood of viral suppression compared with those initiating on an NNTRI [HOR $=0.79(0.75,0.83)]$ and immunologic response decreased with increasing age $[18-<30$ years: ref; $30-<40$ years: aHOR $=0.91(0.84,0.98) ; 40-<50$ years: $\mathrm{aHOR}=0.86(0.80,0.93) ; 50-<60$ years: aHOR=0.90 $(0.82,0.99) ; \geq 60:$ aHOR=0.81 $(0.71,0.92)]$. Age did not modify the effect of initial regime on virologic or immunologic response $(P=0.11$ and $P=0.35$, respectively).

\section{Discussion}

This is the largest study of the impact of age and regimen class on virologic and immunologic response to HAART to date. Our study has several important findings. First, half $(50 \%)$ of individuals who initiated therapy discontinued or changed their initial regimen within the first 2 years. Individuals initiating using an NNRTI-based regimen were more likely to achieve virologic suppression (HIV-1 RNA $\leq 500$ copies/ml) than those initiating using a boosted protease inhibitor-based regimen; however, we did not see a significant increase in virologic suppression with increasing age. In contrast to the virologic outcome, the immunologic outcome did not differ by regimen class, but was clearly impacted by age with older individuals less likely to have an increase in of at least 100 cells $/ \mu 1$. Age at HAART initiation did not modify the effect of the initial regimen on virologic or immunologic response. 
The estimation of 50\% of individuals discontinuing or changing their initial HAART regimen is an underestimation of the proportion that discontinues, or changes, his/her initial HAART regimen in the first 2 years because our inclusionary criteria required that an individual must continue their initial regimen for at least 1 month. Notably, 25\% changed regimen class, suggesting potential side-effect or toxicity, whereas $25 \%$ discontinued treatment for at least 1 month suggesting potential nonadherence. We found a greater proportion of individuals who initiated HAART using a boosted protease inhibitor-based regimen changed regimen class or discontinued treatment compared with individuals initiating using an NNRTI-based regimen (57 and 48\%, respectively). Similarly, in a randomized trial with immunologic outcomes, the proportion that discontinued or changed from their initial HAART regimen class was 43 and 32\% for those who initiated using protease inhibitor and NNRTI regimens, respectively [25]. This could be because protease inhibitors are more difficult to tolerate than NNRTIs with side-effects including diarrhea, hyperlipidemia and glucose dysmetabolism. Studies examining time to toxicity outcomes by age and HAART regimen class are needed to further assess the need for age-specific treatment guidelines.

Our data did not show improved virologic response in older patients compared with younger age groups, as been previously shown [4-12]. Previous studies concluded improved virologic response among older adults was owing to better adherence to HAART, and Silverberg et al. [5], found that controlling for adherence mitigated the differences in virologic responses between age groups [5,20]. Although we were unable to measure and directly account for adherence in our study, our inclusionary criteria and censoring at the time of discontinuation of, or change from, initial HAART regimen resulted in a study population that is more likely to be adherent, regardless of age. Our study criteria for participation and our methods to account for changes in initial HAART regimen were necessary to achieve our objective of estimating the average causal effect of initial HAART regimens on immunologic and virologic response by age. Our findings were similar to the findings of a randomized-controlled trial of viral suppression by initial ART regimen at 96 weeks, with those initiating using NNRTIs more likely to suppress compared with those initiating using boosted protease inhibitors [24].

CD4 cell recovery has been shown to be inversely associated with age and other groups have also found a depressed immunologic response in older patients [12,31,32]. Several mechanisms may account for the suboptimal immunologic response in older individuals, including reduced T-cell regenerative capacity owing to either thymic dysfunction and/or the exhaustion of stem cells, naive $\mathrm{T}$ cells or central memory $\mathrm{T}$ cells. Chronic inflammation may also affect T-cell regeneration. The fact that our findings did not differ by regimen class provides additional evidence for a more broadly defined mechanism for the impact of age on immunologic response to HAART. Although further research is needed to determine if guidelines for when to start HAART should differ by age, our observation that older individuals are less likely to have a robust immunologic response suggest that the optimal time to start HAART might differ by age.

Although a few studies have looked at the effect of regimen on clinical response by age group [5,12], no study has examined the effect of regimen type on clinical response in a population this large or heterogeneous. Bartlett et al. [33], suggested NNRTI-based regimens were associated with improved virologic suppression compared with protease inhibitor-based regimens, but did not comment on how this effect might vary with age. Patterson et al. [11], found immune reconstitution and viral suppression did not vary by treatment regimen when stratified by age. Our results demonstrate an increase in virologic response with NNRTI-based regimens compared with protease inhibitor-based regimens, regardless of age. Although this may suggest that NNRTIs might be preferred in this older 
group, efavirenz, the current guideline-preferred NNRTI, has known CNS adverse effects that also might cause it to be a less desirable drug in some older patients. Studies examining time to side-effect and comorbidity events by age and initial HAART regimen class are needed to further assess the need for age-specific treatment guidelines.

Strengths of this study include the large study population of antiretroviral therapy naïve adults coupled with modern analytical methods to account for the possible selection biased induced by censoring individuals once they discontinued or changed their initial HAART regimen. We did not have data on time from seroconversion to distinguish those who were infected recently from those who have aged with HIV. Survival is suspected to be the driver of the greater proportions of HIV-infected individuals who are older, but it is not the only mechanism. Theoretically, older individuals with HIV infection may have had their infection longer than younger individuals, and these differences may have contributed to the agerelated effects on immunologic response. Another limitation was using a definition of viral suppression $(<500$ copies $/ \mathrm{ml})$ that is higher than current standards $(<200$ copies $/ \mathrm{ml})$; however, owing to the changes in the lower limit detection of HIV-1 RNA assays during our study time period, using a lower limit of detection cut-off of 200 copies $/ \mathrm{ml}$ resulted in omitting 115050 (8\%) viral load measurements, creating a bias by removing many of the participants from the earlier years of our study. Additionally, we did not have data on the reason for the switch or discontinuation. Reasons for switching are important for future studies that could examine differences in toxicity by age and regimen. Lastly, we did not have a direct measurement of adherence, such as drug plasma levels; however, we suspect our study criteria and design restricted the study population to the more adherent HIVinfected individuals.

HIV is a chronic disease that requires life-long therapy and optimizing initial HAART regimen is important. Although future studies will need to evaluate the impact of toxicity and non-AIDS-related comorbidities on virologic and immunologic outcomes, our data do not currently support the use of specific HAART regimens for specific age groups. However, given the impact of CD4 cell count on long-term survival, initiating HAART at higher CD4 cell counts for older individuals may be useful given the decreased likelihood of a robust $(\geq 100$ cells $/ \mu \mathrm{l}) \mathrm{CD} 4$ cell response with increasing age.

\section{Acknowledgments}

We are grateful to all patients, physicians, investigators, and staff involved in the NA-ACCORD. This work was supported by grants from the National Institutes of Health: U01-AI069918, U01-AA013566, UO1-AI-35042, 5MO1-RR-00052 (GCRC), UO1-AI-35043, UO1-AI-35039, UO1-AI-35040, UO1-AI-35041, U01-AI38855: ALLRT; U01-AI38858; U01-AI68634; U01-AI68636; AI-69432; AI-69434, UO1-AI-35004, UO1-AI-31834, UO1-AI-34994, UO1-AI-34989, UO1-AI-34993, and UO1-AI-42590, UO1-HD-32632, UL1-RR024131, P30AI27757; K23-AI-61-0320, P30-AI27767, P30-AI50410; RR025747, P30-AI54999, R01-DA04334; R01DA12568, R01-MH54907, R24-AI067039, N02-CP55504; Z01-CP010176, AHQ290-01-0012, K01-AI071754, K24-00432; R01-DA11602, K01-AI071725, R01 AG026250: Kelly Gebo. This work was also supported by the Centers for Disease Control (CDC200-2006-18797), the Canadian Institutes for Health Research (CIHR: TGF-96118; HCP-97105; CBR-86906; CBR-94036; KRS-86251; 169621) and the Canadian Trials Network (project number 242).

NA-ACCORD participating cohorts (representatives):

(1) AIDS Link to the IntraVenous Experience (Gregory D.Kirk)

(2) Adult AIDS Clinical Trials Group Longitudinal Linked Randomized Trials (Constance A. Benson, Ronald J. Bosch, Ann C. Collier)

(3) HAART Observational Medical Evaluation and Research (Robert S. Hogg, Richard Harrigan, Julio Montaner)

(4) HIV Outpatient Study (John T. Brooks, Kate Buchacz) 
(5) HIV Research Network (Kelly A. Gebo)

(6) Johns Hopkins HIV Clinical Cohort (Richard D. Moore)

(7) John T. Carey Special Immunology Unit Patient Care and Research Database, Case Western Reserve University (Benigno Rodriguez)

(8) Kaiser Permanente Northern California (Michael A. Horberg, Michael J. Silverberg)

(9) Longitudinal Study of Ocular complications of AIDS (Jennifer E. Thorne)

(10) Multicenter Hemophilia Cohort Study-II (James J. Goedert)

(11) Multicenter AIDS Cohort Study (Lisa P. Jacobson)

(12) Montreal Chest Institute Immunodeficiency Service Cohort (Marina B. Klein)

(13) Ontario HIV Treatment Network Cohort Study (Sean B. Rourke, Anita R. Rachlis)

(14) Southern Alberta Clinic Cohort (M. John Gill)

(15) Studies of the Consequences of the Protease Inhibitor Era (Steven G, Deeks, Jeffery N. Martin)

(16) University of Alabama at Birmingham 1917 Clinic Cohort (Michael S. Saag, Michael Mugavero, James Willig)

(17) University of North Carolina, Chapel Hill HIV Clinic Cohort (Joseph J. Eron, Sonia Napravnik)

(18) University of Washington HIV Cohort (Mari M. Kitahata and Heidi M. Crane)

(19) Veterans Aging Cohort Study (Amy C. Justice, David Fiellin)

(20) Vanderbilt-Meharry CFAR Cohort (Timothy R. Sterling, Sam Stinette, Peter Rebeiro, David Haas)

(21) Women's Interagency HIV Study (Stephen J. Gange, Kathryn Anastos).

S.G.D. reports receiving grant support from Merck, Gilead, Bristol-Myers Squibb, and Pfizer; M.S.S. reports receiving consulting fees from Ardea Biosciences, Avexa, Boehringer-Ingelheim, Bristol-Myers Squibb, Gilead Sciences, GlaxoSmithKline, Merck, Monogram Biosciences, Pain Therapeutics, Pfizer, Progenics, Tibotec, Tobira Therapeutics, and Vicro and research support from Avexa, Achillion Pharmaceuticals, Boehringer-Ingelheim, Merck, Pfizer, Progenics, and Tibotec; M.J.S. reports receiving grant support from Pfizer and Merck; M.J.G. reports receiving consulting fees from GlaxoSmithKline,Gilead, Abbott, Merck, Boehringer-Ingelheim, Thera,Tibotec, and Pfizer and grant support from GlaxoSmithKline, Abbott, Canadian Institutes of Health Research, Gilead,Tibotec, and Pfizer; M.B.K. reports receiving consulting fees from GlaxoSmithKline, Abbott, Pfizer, and Merck, lecture fees from Abbott, Gilead, Tibotec, Bristol-Myers Squibb, and GlaxoS-mithKline and research support from Canadian Institutes of Health Research/ Fonds de la recherche en santé du Québec, Canadian HIV Trials Network, Ontario HIV Treatment Network, and Schering Plough Canada; K.A.G. reports receiving consulting fees from Tibotec and grant support from Johns Hopkins University Richard Ross Award, and Agency for Healthcare Research and Quality; K.N.A., A.C.J., S.J.G., B.L., E.T. and S.N. declare they have no conflict of interest.

Sources of Support: This work was supported by grants from the National Institutes of Health: U01-AI069918, U01-AA013566, UO1-AI-35042, 5-MO1-RR-00052 (GCRC), UO1-AI-35043, UO1-AI-35039, UO1-AI-35040, UO1-AI-35041, U01-AI38855: ALLRT; U01-AI38858; U01-AI68634; U01-AI68636; AI-69432; AI-69434, UO1AI-35004, UO1-AI-31834, UO1-AI-34994, UO1-AI-34989, UO1-AI-34993, and UO1-AI-42590, UO1-HD-32632, UL1-RR024131, P30-AI27757; K23-AI-61-0320, P30-AI27767, P30-AI50410; RR025747, P30-AI54999, R01DA04334; R01-DA12568, R01-MH54907, R24-AI067039, N02-CP55504; Z01-CP010176, AHQ290-01-0012, K01-AI071754, K24-00432; R01-DA11602, K01-AI071725, R01 AG026250: K.A.G. This work was also supported by the Centers for Disease Control (CDC200-2006-18797), the Canadian Institutes for Health Research (CIHR: TGF-96118; HCP-97105; CBR-86906; CBR-94036; KRS-86251; 169621) and the Canadian Trials Network (project number 242).

\section{References}

1. Luther VP, Wilkin AM. HIV infection in older adults. Clin Geriatr Med. 2007; 23:567-583. [PubMed: 17631234]

2. Centers for Disease Control and Prevention. HIV/AIDS Surveil-lance Report, 2006. US Department of Health and Human Services, Centers for Disease Control and Prevention; Atlanta: 2008.

3. Palella FJ Jr, Delaney KM, Moorman AC, Loveless MO, Fuhrer J, Satten GA, et al. Declining morbidity and mortality among patients with advanced human immunodeficiency virus infection. HIV Outpatient Study Investigators. N Engl J Med. 1998; 338:853-860. [PubMed: 9516219] 
4. Grabar S, Kousignian I, Sobel A, Le BP, Gasnault J, Enel P, et al. Immunologic and clinical responses to highly active antiretroviral therapy over 50 years of age. Results from the French Hospital Database on HIV. AIDS. 2004; 18:2029-2038. [PubMed: 15577624]

5. Silverberg MJ, Leyden W, Horberg MA, DeLorenze GN, Klein D, Quesenberry CP Jr. Older age and the response to and tolerability of antiretroviral therapy. Arch Intern Med. 2007; 167:684-691. [PubMed: 17420427]

6. Collaboration of Observational HIV Epidemiologic Research Europe (COHERE) Study Group. Response to combination antiretroviral therapy: variation by age. AIDS. 2008; 22:1463-1473. [PubMed: 18614870]

7. Knobel H, Guelar A, Valldecillo G, Carmona A, Gonzalez A, Lopez-Colomes JL, et al. Response to highly active antiretroviral therapy in HIV-infected patients aged 60 years or older after 24 months follow-up. AIDS. 2001; 15:1591-1593. [PubMed: 11505000]

8. Fleishman, JA. Disparities in virologic suppression among patients with HIV infection. Academy Health Annual Research Meeting; San Diego, CA. 2004.

9. Goodkin K, Shapshak P, Asthana D, Zheng W, Concha M, Wilkie FL, et al. Older age and plasma viral load in HIV-1 infection. AIDS. 2004; 18:S87-S98. [PubMed: 15075503]

10. Tumbarello M, Rabagliati R, de Gaetano DK, Bertagnolio S, Montuori E, Tamburrini E, et al. Older age does not influence CD4 cell recovery in HIV-1 infected patients receiving highly active antiretroviral therapy. BMC Infect Dis. 2004; 4:46. [PubMed: 15530169]

11. Patterson K, Napravnik S, Eron J, Keruly J, Moore R. Effects of age and sex on immunological and virological responses to initial highly active antiretroviral therapy. HIV Med. 2007; 8:406410. [PubMed: 17661850]

12. Greenbaum AH, Wilson LE, Keruly JC, Moore RD, Gebo KA. Effect of age and HAART regimen on clinical response in an urban cohort of HIV-infected individuals. AIDS. 2008; 22:2331-2339. [PubMed: 18981772]

13. Manfredi R, Chiodo F. A case-control study of virological and immunological effects of highly active antiretroviral therapy in HIV-infected patients with advanced age. AIDS. 2000; 14:14751477. [PubMed: 10930176]

14. Viard JP, Mocroft A, Chiesi A, Kirk O, Roge B, Panos G, et al. Influence of age on CD4 cell recovery in human immunodeficiency virus-infected patients receiving highly active antiretroviral therapy: evidence from the EuroSIDA study. J Infect Dis. 2001; 183:1290-1294. [PubMed: 11262215]

15. Kalayjian RC, Landay A, Pollard RB, Taub DD, Gross BH, Francis IR, et al. Age-related immune dysfunction in health and in human immunodeficiency virus (HIV) disease: association of age and HIV infection with naive CD8R cell depletion, reduced expression of CD28 on CD8R cells, and reduced thymic volumes. J Infect Dis. 2003; 187:1924-1933. [PubMed: 12792869]

16. Cuzin L, Delpierre C, Gerard S, Massip P, Marchou B. Immunologic and clinical responses to highly active antiretroviral therapy in patients with HIV infection aged $>50$ years. Clin Infect Dis. 2007; 45:654-657. [PubMed: 17683004]

17. Yamashita TE, Phair JP, Munoz A, Margolick JB, Detels R, O'Brien SJ, et al. Immunologic and virologic response to highly active antiretroviral therapy in the Multicenter AIDS Cohort Study. AIDS. 2001; 15:735-746. [PubMed: 11371688]

18. Grimes RM, Otiniano ME, Rodriguez-Barradas MC, Lai D. Clinical experience with human immunodeficiency virus-infected older patients in the era of effective antiretroviral therapy. Clin Infect Dis. 2002; 34:1530-1533. [PubMed: 12015701]

19. Tumbarello M, Rabagliati R, de Gaetano DK, Bertagnolio S, Tamburrini E, Tacconelli E, et al. Older HIV-positive patients in the era of highly active antiretroviral therapy: changing of a scenario. AIDS. 2003; 17:128-131. [PubMed: 12478081]

20. Hinkin CH, Hardy DJ, Mason KI, Castellon SA, Durvasula RS, Lam MN, et al. Medication adherence in HIV-infected adults: effect of patient age, cognitive status, and substance abuse. AIDS. 2004; 18:S19-S25. [PubMed: 15075494]

21. Manfredi R, Calza L, Cocchi D, Chiodo F. Antiretroviral treatment and advanced age: epidemiologic, laboratory, and clinical features in the elderly. J Acquir Immune Defic Syndr. 2003; 33:112-114. [PubMed: 12792363] 
22. Panel on antiretroviral guidelines for adults and adolescents. Guidelines for the use of antiretroviral agents in HIV-1-infected adults and adolescents. 2008. p. 1-139.

23. Hammer SM, Eron JJ Jr, Reiss P, Schooley RT, Thompson MA, Walmsley S, et al. Antiretroviral treatment of adult HIV infection: 2008 recommendations of the International AIDS Society-USA panel. JAMA. 2008; 300:555-570. [PubMed: 18677028]

24. Riddler SA, Haubrich R, DiRienzo AG, Peeples L, Powderly WG, Klingman KL, et al. Classsparing regimens for initial treatment of HIV-1 infection. N Engl J Med. 2008; 358:2095-2106. [PubMed: 18480202]

25. MacArthur RD, Novak RM, Peng G, Chen L, Xiang Y, Hullsiek KH, et al. A comparison of three highly active antiretroviral treatment strategies consisting of nonnucleoside reverse transcriptase inhibitors, protease inhibitors, or both in the presence of nucleoside reverse transcriptase inhibitors as initial therapy (CPCRA 058 FIRST Study): a long-term randomised trial. Lancet. 2006; 368:2125-2135. [PubMed: 17174704]

26. Gange SJ, Kitahata MM, Saag MS, Bangsberg DR, Bosch RJ, Brooks JT, et al. Cohort profile: the North American AIDS Cohort Collaboration on Research and Design (NA-ACCORD). Int J Epidemiol. 2007; 36:294-301. [PubMed: 17213214]

27. Cuzick J, Edwards R, Segnan N. Adjusting for noncompliance and contamination in randomized clinical trials. Stat Med. 1997; 16:1017-1029. [PubMed: 9160496]

28. Cain LE, Cole SR. Inverse probability-of-censoring weights for the correction of time-varying noncompliance in the effect of randomized highly active antiretroviral therapy on incident AIDS or death. Stat Med. 2009; 28:1725-1738. [PubMed: 19347843]

29. Hernan MA, Lanoy E, Costagliola D, Robins JM. Comparison of dynamic treatment regimes via inverse probability weighting. Basic Clin Pharmacol Toxicol. 2006; 98:237-242. [PubMed: 16611197]

30. Cole SR, Hernan MA. Adjusted survival curves with inverse probability weights. Comput Methods Programs Biomed. 2004; 75:45-49. [PubMed: 15158046]

31. Belanger F, Meyer L, Carre N, Coutellier A, Deveau C. Influence of age at infection on human immunodeficiency virus disease progression to different clinical endpoints: the SEROCO cohort (1988-1994). The Seroco Study Group. Int J Epidemiol. 1997; 26:1340-1345. [PubMed: 9447415]

32. Munoz A, Sabin CA, Phillips AN. The incubation period of AIDS. AIDS. 1997; 11:S69-S76. [PubMed: 9451969]

33. Bartlett, J.; Johnson, J.; Herrera, J. Initial therapy with abacavir+lamivudine (ABCR3TC) combined with efavirenz (NNRTI), amprenavir/ritonavir (PI) or stavudine (NRTI): ESS40001 (CLASS). XVth International AIDS Conference; Bangkok. 2009. 

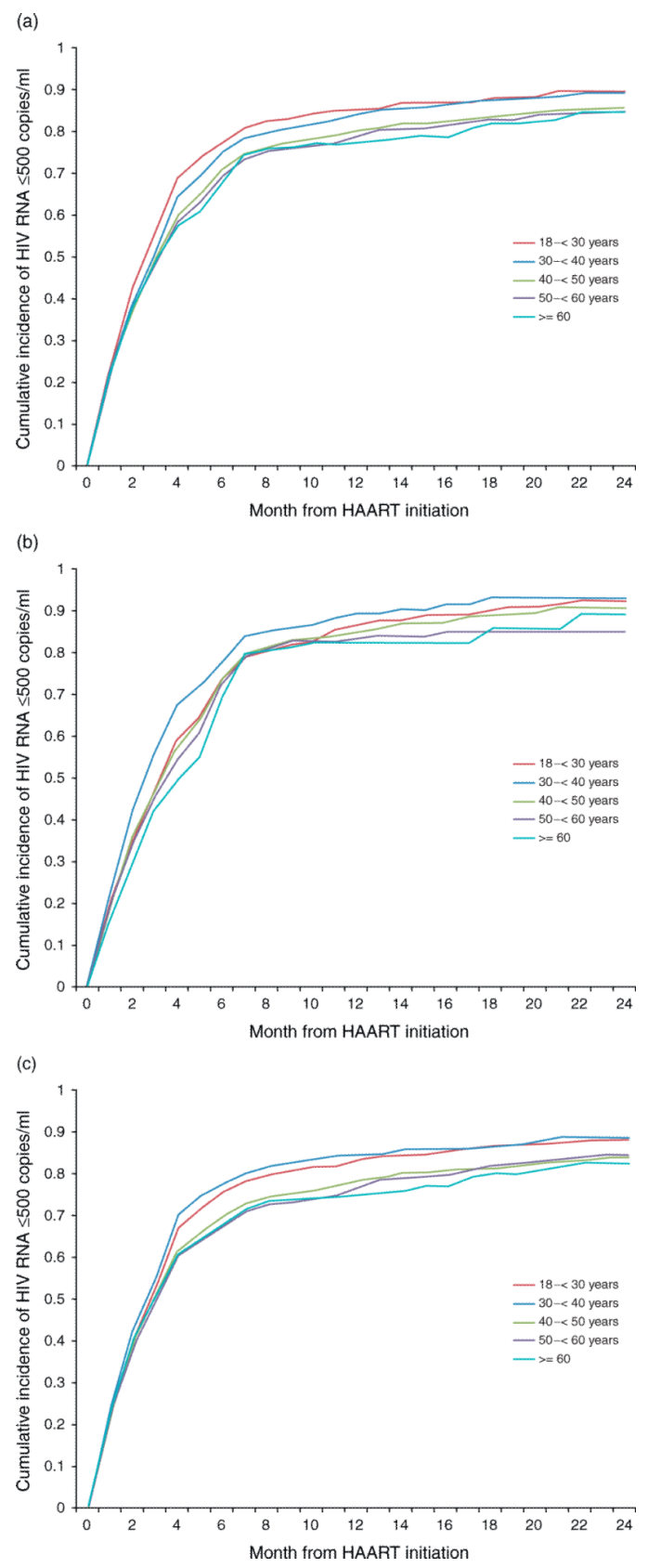

Fig. 1. Cumulative incidence of HIV RNA at least 500 copies/ml in the first 2 years after HAART initiation, by age group and initial HAART regimen class

(a) Overall cumulative incidence of HIV RNA 500 copies $/ \mathrm{ml}$ or less in the first 2 years after HAART initiation. (b) Cumulative incidence of HIV RNA 500 copies/ml or less in those initiating HAART with a boosted PI-based regimen. (c) Cumulative incidence of HIV RNA 500 copies/ml or less in those initiating HAART with an NNRTI-based regimen. HAART highly active antiretroviral treatment, NNRTI nonnucleoside reverse transcriptase inhibitor, PI protease inhibitor. 
(a)

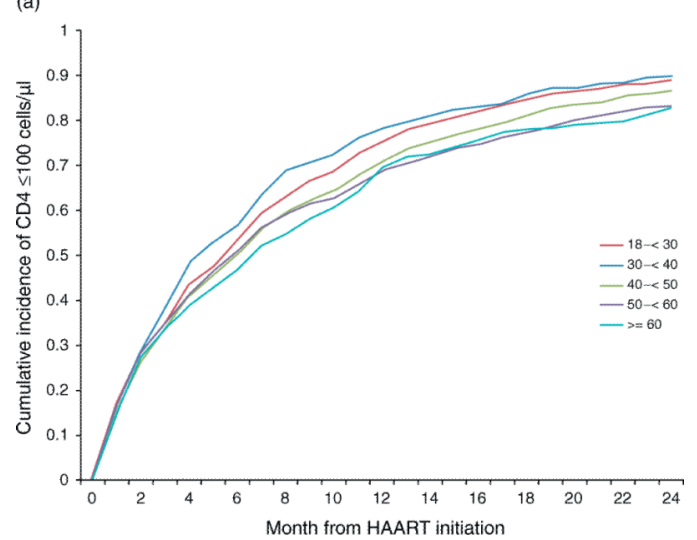

(b)

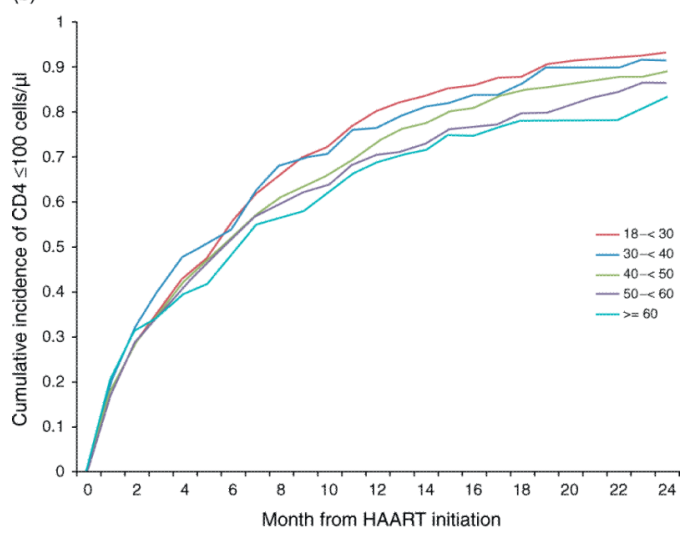

(c)

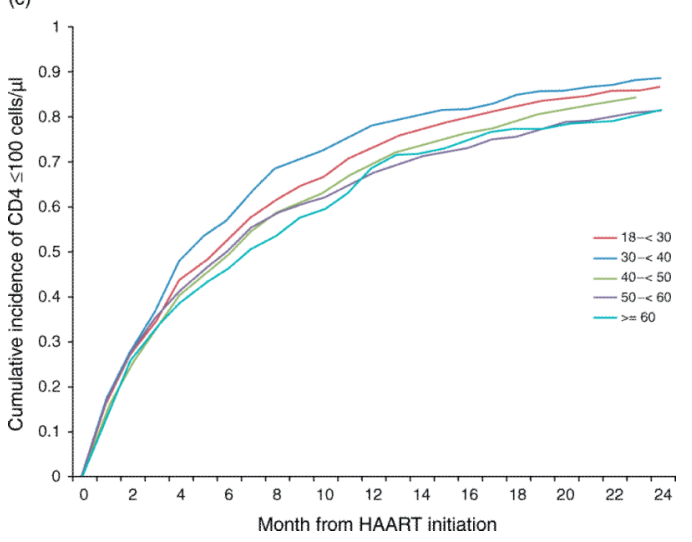

Fig. 2. Cumulative incidence of an increase in CD4 cells of at least 100 cells/ml in the first 2 years after HAART initiation, by age group and initial HAART regimen class

(a) Overall cumulative incidence of at least 100 cells $/ \mathrm{ml}$ in the first 2 years after HAART initiation. (b) Cumulative incidence of an increase of at least 100 cells $/ \mathrm{ml}$ in those initiating HAART with a boosted PI-based regimen. (c) Cumulative incidence of an increase of at least 100 cells $/ \mathrm{ml}$ in those initiating HAART with NNRTI-based regimen. NNRTI nonnucleoside reverse transcriptase inhibitor, PI protease inhibitor. 


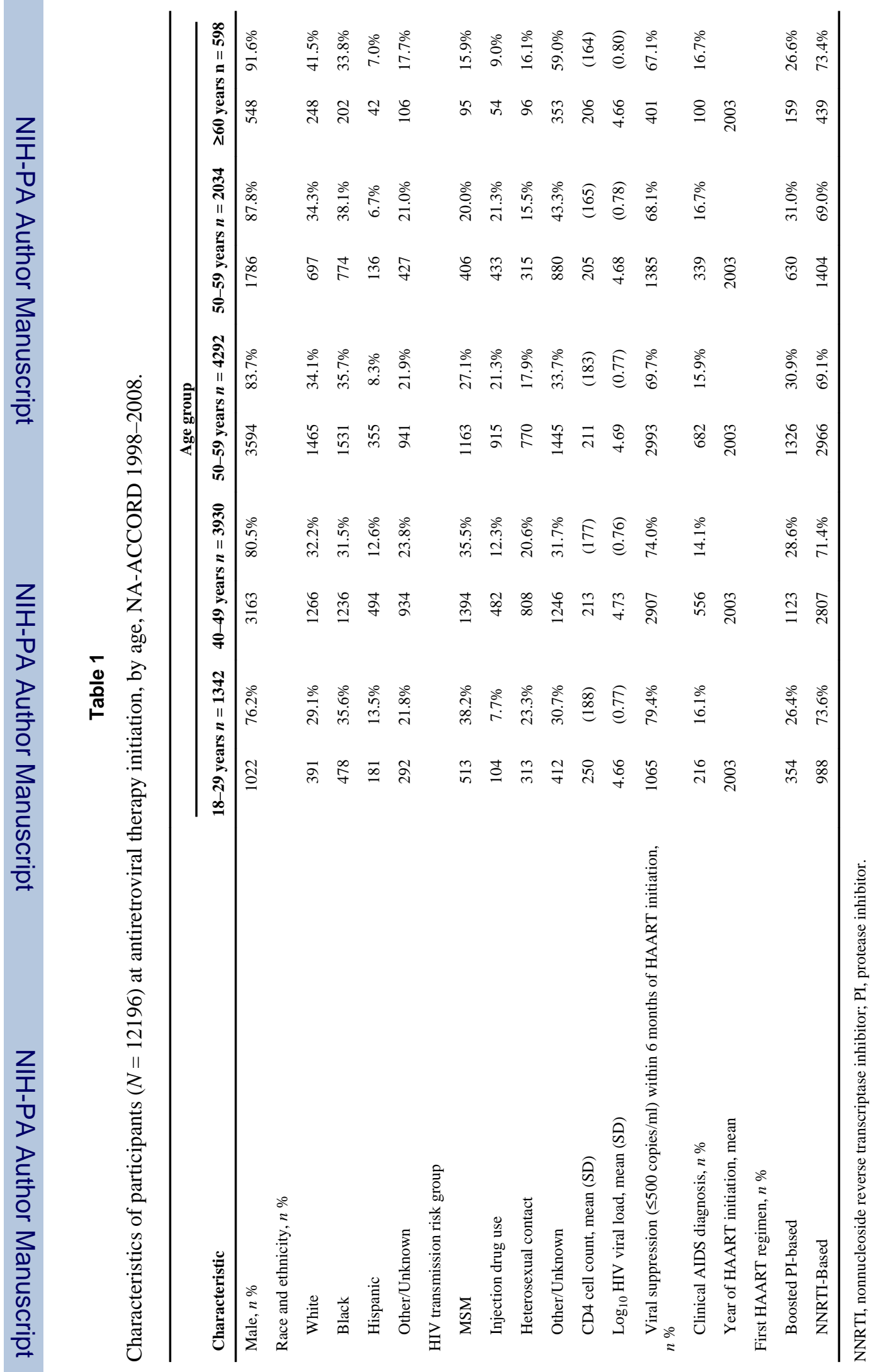


Table 2

Adjusted $^{a}$ hazard odds ratios (aHOR) and 95\% confidence intervals (95\% CI) of virologic and immunologic response to HAART within 24 months following HAART initiation, NA-ACCORD, 1998-2008.

\begin{tabular}{|c|c|c|c|c|}
\hline & $\begin{array}{c}\text { Outcomes HIV-1 RNA } \\
\text { suppression }(<500 \text { copies/ml) }\end{array}$ & & $\begin{array}{l}\text { CD4 cell count increase of } 100 \\
\text { cells } / \mu \mathrm{l}\end{array}$ & \\
\hline & $\mathrm{aHOR}^{a}$ & $95 \% \mathrm{CI}$ & $\mathrm{aHOR}^{a}$ & $95 \% \mathrm{CI}$ \\
\hline \multicolumn{5}{|l|}{ Age (years) } \\
\hline $18-<30$ & REF & - & REF & - \\
\hline $30-<40$ & 0.71 & $0.89-1.05$ & 0.92 & $0.85-1.00$ \\
\hline $40-<50$ & 0.66 & $0.89-1.04$ & 0.85 & $0.78-0.92$ \\
\hline $50-<60$ & 1.00 & $0.91-1.10$ & 0.82 & $0.74-0.90$ \\
\hline$\geq 60$ & 1.05 & $0.92-1.20$ & 0.74 & $0.65-0.85$ \\
\hline \multicolumn{5}{|l|}{ Initial HAART regimen class } \\
\hline NNRTI & REF & - & REF & - \\
\hline Boosted PI & 0.77 & $0.73-0.82$ & 1.03 & $0.98-1.09$ \\
\hline \multicolumn{5}{|l|}{ CD4 cell count at HAART initiation } \\
\hline$<350$ cells $/ \mu 1$ & REF & - & REF & - \\
\hline$\geq 350$ cells $/ \mu 1$ & 0.94 & $0.89-1.00$ & 1.05 & $0.98-1.12$ \\
\hline $\log _{10}$ HIVRNA at HAART initiation & 0.84 & $0.82-0.87$ & 1.32 & $1.27-1.36$ \\
\hline \multicolumn{5}{|l|}{ Sex } \\
\hline Male & REF & - & REF & - \\
\hline Female & 0.98 & $0.92-1.05$ & 1.13 & $1.05-1.21$ \\
\hline \multicolumn{5}{|l|}{ Race and ethnicity } \\
\hline White & REF & - & $\mathrm{REF}$ & - \\
\hline Black & 0.91 & $0.86-0.97$ & 0.87 & $0.82-0.92$ \\
\hline Hispanic & 0.94 & $0.86-1.02$ & 0.87 & $0.79-0.95$ \\
\hline Other/Unknown & 0.99 & $0.92-1.06$ & 0.92 & $0.86-1.00$ \\
\hline \multicolumn{5}{|l|}{ HIV transmission risk group } \\
\hline MSM & REF & - & REF & - \\
\hline Injection drug use & 0.85 & $0.78-0.93$ & 0.78 & $0.71-0.86$ \\
\hline Heterosexual contact & 0.92 & $0.85-0.99$ & 0.86 & $0.79-0.93$ \\
\hline Other/unknown & 0.88 & $0.81-0.96$ & 0.88 & $0.81-0.96$ \\
\hline \multicolumn{5}{|l|}{ Clinical AIDS prior to HAART initiation } \\
\hline No & REF & - & REF & - \\
\hline Yes & 1.04 & $0.97-1.11$ & 0.94 & $0.88-1.01$ \\
\hline \multicolumn{5}{|l|}{ Year of HAART initiation } \\
\hline 1998 & REF & - & REF & - \\
\hline 1999 & 1.16 & $0.99-1.37$ & 0.92 & $0.78-1.08$ \\
\hline 2000 & 1.21 & $1.03-1.42$ & 0.93 & $0.79-1.09$ \\
\hline 2001 & 1.32 & $1.13-1.55$ & 0.89 & $0.76-1.05$ \\
\hline 2002 & 1.15 & $0.98-1.34$ & 0.91 & $0.78-1.07$ \\
\hline 2003 & 1.30 & $1.11-1.52$ & 1.03 & $0.88-1.21$ \\
\hline
\end{tabular}




\begin{tabular}{lcccc}
\hline & $\begin{array}{c}\text { Outcomes HIV-1 RNA } \\
\text { suppression }(<\mathbf{5 0 0} \text { copies/ml) }\end{array}$ & \multicolumn{2}{c}{$\begin{array}{c}\text { CD4 cell count increase of 100 } \\
\text { cells/ } \mathbf{\mu l}\end{array}$} \\
\hline 2004 & 1.41 & $1.20-1.65$ & 0.99 & $0.84-1.17$ \\
2005 & 1.43 & $1.22-1.68$ & 1.13 & $0.96-1.34$ \\
2006 & 1.55 & $1.32-1.83$ & 1.14 & $0.97-1.35$ \\
2007 & 2.02 & $1.70-2.40$ & 1.21 & $1.02-1.44$ \\
2008 & 2.32 & $1.93-2.78$ & 1.39 & $1.15-1.67$ \\
\hline
\end{tabular}

AIDS acquired immune deficiency syndrome, HAART highly active antiretroviral treatment, HIV human immunodeficiency virus, NNRTI nonnucleoside reverse transcriptase inhibitor, PI protease inhibitor.

${ }^{a}$ Pooled logistic regression models weighted for the first change in ART regimen class (weights at the 1st and 99th percentile were truncated) and adjusted for cohort. 
Table 3

Adjusted $^{a}$ hazard odds ratios (aHOR) and $95 \%$ confidence intervals (95\% CI) virologic and immunologic response to HAART within 24 months following HAART initiation, by regimen class and age, NAACCORD, 1998-2008.

\begin{tabular}{|c|c|c|c|c|}
\hline & aHOR $^{a}$ NNRTI & $95 \% \mathrm{CI}$ & aHOR $^{a}$ Boosted PI & $95 \% \mathrm{CI}$ \\
\hline \multicolumn{5}{|c|}{ Virologic response (HIV RNA $\leq 500$ copies/ml) } \\
\hline \multicolumn{5}{|c|}{ Age (years) } \\
\hline $18-<30$ & Ref & - & 0.75 & $0.64-0.88$ \\
\hline $30-<40$ & 0.98 & $0.89-1.07$ & 0.71 & $0.64-0.79$ \\
\hline $40-<50$ & 0.94 & $0.85-1.03$ & 0.76 & $0.68-0.85$ \\
\hline $50-<60$ & 0.98 & $0.88-1.09$ & 0.79 & $0.69-0.90$ \\
\hline$\geq 60$ & 1.06 & $0.91-1.24$ & 0.77 & $0.63-0.94$ \\
\hline \multicolumn{5}{|c|}{ Immunologic response ( $\geq 100$ cells $/ \mu 1$ increase in $C D 4$ cell count) } \\
\hline \multicolumn{5}{|l|}{ Age (years) } \\
\hline $18-<30$ & Ref & - & 0.94 & $0.80-1.11$ \\
\hline $30-<40$ & 0.90 & $0.82-0.99$ & 0.96 & $0.86-1.07$ \\
\hline $40-<50$ & 0.83 & $0.76-0.91$ & 0.88 & $0.79-0.99$ \\
\hline $50-<60$ & 0.80 & $0.72-0.90$ & 0.84 & $0.73-0.96$ \\
\hline$\geq 60$ & 0.73 & $0.63-0.85$ & 0.76 & $0.60-0.97$ \\
\hline
\end{tabular}

AIDS acquired immune deficiency syndrome, HAART highly active antiretroviral treatment, HIV human immunodeficiency virus, NNRTI nonnucleoside reverse transcriptase inhibitor, PI protease inhibitor.

${ }^{a}$ Pooled logistic regression models weighted for the first change in HAART regimen class (weights at the 1st and 99th percentile were truncated) and adjusted for CD4 cell and HIV viral load at initiation, race and ethnicity, HIV transmission risk group, sex, cohort, clinical AIDS diagnosis prior to HAART initiation, year of HAART initiation and month from HAART initiation. 